\title{
Maternal and fetal blood lipid concentrations during pregnancy differ by maternal body mass index: findings from the ROLO study
}

Aisling A. Geraghty ${ }^{1}$, Goiuri Alberdi ${ }^{1}$, Elizabeth J. O'Sullivan ${ }^{1}$, Eileen C. O'Brien ${ }^{1}$, Brenda Crosbie², Patrick J. Twomey ${ }^{2,3}$ and Fionnuala M. McAuliffe

\begin{abstract}
Background: Pregnancy is a time of altered metabolic functioning and maternal blood lipid profiles change to accommodate the developing fetus. While these changes are physiologically necessary, blood lipids concentrations have been associated with adverse pregnancy outcomes such as gestational diabetes, pregnancy-induced hypertension and high birth weight. As blood lipids are not routinely measured during pregnancy, there is limited information on what is considered normal during pregnancy and in fetal blood.

Methods: Data from 327 mother-child pairs from the ROLO longitudinal birth cohort study were analysed. Fasting total cholesterol and triglycerides were measured in early and late pregnancy and fetal cord blood. Intervals were calculated using the 2.5th, 50th and 97.5th centile. Data was stratified based on maternal body mass index (BMI) measured during early pregnancy. Differences in blood lipids between BMI categories were explored using ANOVA and infant outcomes of macrosomia and large-for-gestational-age (LGA) were explored using independent student T-tests and binary logistic regression.
\end{abstract}

Results: All maternal blood lipid concentrations increased significantly from early to late pregnancy. In early pregnancy, women with a BMl $<25 \mathrm{~kg} / \mathrm{m}^{2}$ had lower concentrations of total cholesterol compared to women with a BMl of 25-29. $9 \mathrm{~kg} / \mathrm{m}^{2}(P=0.02)$. With triglycerides, women in the obese category $\left(\mathrm{BMl}>30 \mathrm{~kg} / \mathrm{m}^{2}\right)$ had higher concentrations than both women in the normal-weight and overweight category in early and late pregnancy $(P<0.001$ and $P=0.03$, respectively). In late pregnancy, triglyceride concentrations remained elevated in women in the obese category compared to women in the normal-weight category $(P=0.01)$. Triglyceride concentrations were also elevated in late pregnancy in mothers that then gave birth to infants with macrosomia and LGA ( $P=0.01$ and $P=0.03$, respectively).

Conclusion: Blood lipid concentrations increase during pregnancy and differ by maternal BMI. These intervals could help to inform the development of references for blood lipid concentrations during pregnancy.

Trial registration: ROLO Study - ISRCTN54392969. Date of registration: 22/04/2009.

Keywords: Pregnancy, Lipids, Cholesterol, References, Cord blood, BMl, Health

\footnotetext{
* Correspondence: fionnuala.mcauliffe@ucd.ie

${ }^{1}$ UCD Perinatal Research Centre, Obstetrics and Gynaecology, School of

Medicine, University College Dublin, National Maternity Hospital, Dublin 2,

Ireland

Full list of author information is available at the end of the article
} 


\section{Background}

Pregnancy is a unique physiological state that results in alterations in the mother's metabolic functioning. These alterations ensure that both the mother and fetus have adequate energy stores throughout pregnancy, and ensure appropriate development of the fetus. As the fetoplacental unit requires glucose, amino acids and lipids throughout pregnancy, the mother's metabolism must adapt to ensure that this supply is met. These adaptations influence maternal blood lipid concentrations and during pregnancy mothers often enter a physiologically-normal state of hyperlipidemia [1-3]. After delivery, lipid concentrations return to prepregnancy concentrations which suggests that this rise in blood lipids could play a role in the physiology of the pregnancy and the development of the fetus [3].

However, raised blood lipid concentrations, above those considered normal during pregnancy, have been associated with negative health outcomes for both the mother and the child. Research suggests that earlypregnancy lipid profiles, particularly elevated total cholesterol and triglyceride concentrations, may be used to identify those at increased risk of developing gestational diabetes [4-6]. Elevated triglyceride concentrations have also been associated with increased risk of pregnancyinduced hypertension and pre-eclampsia in a European cohort [7]. Research has also shown that metabolic changes in pregnancy may be related to the composition of lipids and lipoproteins [8]. This may influence the flux of lipids to the placenta which may, in turn, alter fetal lipid concentrations.

In relation to infant outcomes, maternal triglycerides have been positively associated with fetal size, birth weight and fat mass [9]. Higher total cholesterol and triglyceride concentrations have been associated with higher birth weight [10], increased risk of large-for-gestational age (LGA) [7, 11], and pre-term delivery [7]. However, the point at which maternal blood lipid concentrations become problematic for maternal and infant health outcomes is unclear. One study examined associations of maternal total cholesterol concentrations and fetoplacental endothelial dysfunction and cited above the 75th percentile value $(7.5 \mathrm{mmol} / \mathrm{L})$ in their cohort as abnormal, based on observations of endothelial dysfunction above this concentration [12]. There is potential for the use of maternal triglyceride concentrations as predictive factors for LGA births in women with gestational diabetes [13].

Additionally, there is a paucity of data available on fetal blood lipid concentrations. One study identified lipid profiles that differed in small-for-gestational-age infants compared to normal-weight infants, with increased levels of apo C-I-enriched high-density lipoproteins [14], however information is still lacking in this area. Maternal lipid concentrations have been correlated with fetal lipid concentrations at birth among women with diabetes, which highlights the importance of the maternal environment on the fetus during pregnancy [9].

Recommended blood lipid concentrations are available for non-pregnant individuals; below $5 \mathrm{mmol} / \mathrm{L}$ for total cholesterol and below $2 \mathrm{mmol} / \mathrm{L}$ for triglycerides [15]. However, there are currently no recommended intervals available for maternal blood lipid concentrations during pregnancy or for fetal blood. Thus, the aim of this paper was to address this knowledge-deficit by describing blood lipid concentrations in our cohort of healthy women with singleton pregnancies without gestational diabetes or pre-eclampsia. While these women had all previously given birth to macrosomic infants, the cohort had no complications during pregnancy. As research has shown that blood lipid concentrations differ by maternal BMI [16, 17], intervals were stratified by maternal BMI category.

\section{Methods \\ Study design}

A group of 327 mother and child dyads with blood lipid measurements available from during pregnancy from the ROLO (Randomised cOntrol trial of LOw glycaemic index diet) study were included in this analysis. The original study involved 800 secundigravida women, whose first baby had a birth weight above $4 \mathrm{~kg}$, randomised to either a low glycaemic index diet during pregnancy or routine antenatal natal care with no specific dietary advice. This study was carried out in The National Maternity Hospital, Dublin, Ireland, with institutional ethical approval and maternal written consent. Trial registration number for the ROLO study is ISRCTN54392969. Detailed methods and results of the ROLO study have previously been published [18-20]. In brief, the intervention involved one dietary education session (and two follow-up meetings at 28 and 34 weeks' gestation) with a research dietitian where the women were educated about the glycaemic index and encouraged to follow a low glycaemic index diet. The recommended diet was eucaloric and the women were not advised to reduce their total energy intake. The intervention did not influence birth weight, however, mothers in the intervention group had significantly less gestational weight gain and improved glucose tolerance [21]. The intervention had no impact on maternal or fetal blood lipid profiles so all participants are analysed together in this analysis. The women included all had healthy, full-term singleton pregnancies; there were no adverse maternal outcomes in pregnancy, such as gestational diabetes or pre-eclampsia, and no pre-term births. 


\section{Data collection}

Height and weight were measured by trained professionals in early pregnancy (at 13 weeks' gestation, as calculated based on the participant's last menstrual period and first trimester ultrasound) in the hospital, and BMI $\left(\mathrm{kg} / \mathrm{m}^{2}\right)$ was calculated. Maternal overnight fasting blood samples were taken in early pregnancy and late pregnancy (at 28 weeks' gestation) and a fetal cord blood serum sample was collected at delivery. Birth weight was recorded at delivery along with incidence of macrosomia (birth weight of or above $4 \mathrm{~kg}$ ) and LGA, which was classified as birth weight above the 90th centile (taking into account gestational age and sex).

\section{Laboratory analyses}

Serum total cholesterol was measured using Roche cholesterol oxidase method and triglyceride concentrations were measured using the lipase/GPO-PAP (glycerol phosphate oxidase-p-aminophenazone). All measurements were carried out on the cobas c702 module of the Roche Cobas 8000 analyser in accordance with the manufacturer's instructions (Roche Diagnostics GmbH, Penzburg, Germany). The methods are standardised against the isotope dilution/ mass spectrometry (ID/MS) methods.

\section{Statistical analyses}

All variables were evaluated for normal distribution by visual analysis of histograms and non-normal data underwent logarithmic transformation. Antilogarithms were used in the presentation of the results, where required. Intervals were calculated using the 2.5th, 50th and 97.5th centile for the total group and for each BMI category; BMI $<25 \mathrm{~kg} / \mathrm{m}^{2}$, BMI $25-29.9 \mathrm{~kg} / \mathrm{m}^{2}$ and BMI $\geq 30 \mathrm{~kg} / \mathrm{m}^{2}$. Differences between the central tendencies in these categories were examined using one- way ANOVA or Chi-square tests, as appropriate. Correlations between blood lipid concentrations are each time point was examined using Pearson correlation for normally distributed data and Spearman's correlation for non- normal data. Differences in blood cholesterol and triglycerides based on birth outcomes of macrosomia and LGA were examined using independent sample ttests, and binary logistic regression was carried out to investigate the association of maternal BMI on this. Statistical analyses were carried out using SPSS (Statistical Package for the Social Sciences) software version 20.0 (IBM, Armonk, NY).

\section{Results}

\section{Cohort characteristics}

Mean BMI at 13 weeks' gestation was $26.4 \mathrm{~kg} / \mathrm{m}^{2}$, which is in the overweight category (Table 1). Just over half (54.4\%) of the study participants had a BMI $\geq 25 \mathrm{~kg} / \mathrm{m}^{2}$. Mean infant birth weight was $4.06 \mathrm{~kg}$ with $54.7 \%$ of infants classified as macrosomic and $29.3 \%$ as LGA. Mean birth weight centile was 72.9 . There was a significant difference in birth weight with babies born to mothers with a BMI $\geq 25 \mathrm{~kg} / \mathrm{m}^{2}$ having a higher birth weight than those born to mothers with a BMI $<25 \mathrm{~kg} / \mathrm{m}^{2}$ $(P=0.02)$.

The intervals for maternal blood lipids in early and late pregnancy and for fetal cord blood concentrations are described for the total group and each maternal BMI category (Table 2). Both total cholesterol and triglyceride concentrations increased significantly from early pregnancy to late pregnancy $(P<0.001)$. The median concentrations of total cholesterol in early pregnancy, for the total group and each BMI category, are within the recommendations for non-pregnant individuals, below $5 \mathrm{mmol} / \mathrm{L}$, however by late pregnancy $67.7 \%$ of the participants exceeded this concentration. The median

Table 1 Characteristics of mothers and infants from the ROLO Cohort

\begin{tabular}{|c|c|c|c|c|c|}
\hline & Total $(n=327)$ & $\mathrm{BMI}<25(n=149)$ & BMI 25-29.9 $(n=127)$ & $\mathrm{BMI} \geq 30(n=51)$ & $P$-value \\
\hline \multicolumn{6}{|l|}{ Maternal Characteristics } \\
\hline Age at delivery (years) & $33.10(3.90)$ & $33.44(3.75)$ & $32.80(4.10)$ & $32.59(3.91)$ & 0.29 \\
\hline BMl at 13 weeks' gestation $\left(\mathrm{kg} / \mathrm{m}^{2}\right)$ & $26.40(4.60)$ & $22.83(1.61)$ & $27.11(1.40)$ & $34.77(3.65)$ & - \\
\hline Achieved 3rd level education (n(\%)) & $174(58.6)$ & $83(61.0)$ & $71(55.0)$ & $18(40.0)$ & $0.03^{*}$ \\
\hline Smoked during pregnancy $(\mathrm{n}(\%))$ & $7(2.1)$ & $0(0)$ & $6(4.7)$ & $1(2)$ & $0.03^{*}$ \\
\hline Ethnicity (Caucasian) (n(\%)) & $323(98.8)$ & $147(98.6)$ & $127(100)$ & $50(98.0)$ & 0.66 \\
\hline \multicolumn{6}{|l|}{ Neonatal/Infant Characteristics } \\
\hline Male sex $(n(\%))$ & $157(47.4)$ & $74(49.7)$ & $57(44.2)$ & $25(49)$ & 0.64 \\
\hline Gestational age at delivery (weeks) & $40.4(1.07)$ & $40.33(1.07)$ & $40.49(0.98)$ & $40.40(1.32)$ & 0.51 \\
\hline Birth weight (kg) & $4.07(0.47)$ & $4.00(0.47)$ & $4.13(0.44)$ & $4.10(0.55)$ & 0.07 \\
\hline Birth weight centile & $72.90(24.9)$ & $74.36(25.65)$ & $74.27(22.26)$ & $64.91(27.91)$ & 0.06 \\
\hline
\end{tabular}

All data expressed as either mean (SD) or $\mathrm{n}(\%)$ for available data on cohort, BMI Body mass index. Statistical comparisons by one-way ANOVA or Chi-square tests. ${ }^{*} P<0.05$ 
Table 2 Blood lipid Intervals during early and late pregnancy by maternal BMI category

\begin{tabular}{|c|c|c|c|c|c|c|c|c|c|c|c|c|}
\hline \multicolumn{13}{|c|}{ Early Pregnancy (13 weeks' gestation) } \\
\hline & \multicolumn{3}{|c|}{ Total Group $(n=284)$} & \multicolumn{3}{|c|}{$\mathrm{BMI}<25(n=136)$} & \multicolumn{3}{|c|}{ BMI 25-29.99 $(n=104)$} & \multicolumn{3}{|c|}{$\mathrm{BMI} \geq 30(n=42)$} \\
\hline Percentile & 2.5 & 50 & 97.5 & 2.5 & 50 & 97.5 & 2.5 & 50 & 97.5 & 2.5 & 50 & 97.5 \\
\hline Total cholesterol & 1.75 & 4.58 & 6.73 & 1.59 & 4.29 & 6.69 & 1.67 & 4.80 & 6.92 & 1.77 & 4.65 & 6.31 \\
\hline Triglycerides & 0.35 & 1.03 & 2.12 & 0.29 & 0.94 & 2.02 & 0.37 & 1.07 & 2.13 & 0.37 & 1.32 & 2.54 \\
\hline \multicolumn{13}{|c|}{ Late Pregnancy (28 weeks' gestation) } \\
\hline & \multicolumn{3}{|c|}{ Total Group $(n=293)$} & \multicolumn{3}{|c|}{$\mathrm{BMI}<25(\mathrm{n}=136)$} & \multicolumn{3}{|c|}{ BMI 25-29.9 $(n=114)$} & \multicolumn{3}{|c|}{$\mathrm{BMI} \geq 30(n=41)$} \\
\hline Percentile & 2.5 & 50 & 97.5 & 2.5 & 50 & 97.5 & 2.5 & 50 & 97.5 & 2.5 & 50 & 97.5 \\
\hline Total cholesterol & 2.1 & 6.02 & 8.61 & 1.83 & 6.06 & 8.82 & 2.41 & 6.11 & 8.57 & 1.97 & 5.63 & 8.50 \\
\hline Triglycerides & 0.58 & 1.71 & 3.24 & 0.40 & 1.66 & 2.91 & 0.61 & 1.71 & 3.33 & 0.64 & 1.84 & 5.12 \\
\hline \multicolumn{13}{|c|}{ Fetal Cord Blood (delivery) } \\
\hline & \multicolumn{3}{|c|}{ Total Group $(n=227)$} & \multicolumn{3}{|c|}{$\mathrm{BMI}<25(n=97)$} & \multicolumn{3}{|c|}{ BMI 25-29.9 $(n=96)$} & \multicolumn{3}{|c|}{$\mathrm{BMI} \geq 30(n=33)$} \\
\hline Percentile & 2.5 & 50 & 97.5 & 2.5 & 50 & 97.5 & 2.5 & 50 & 97.5 & 2.5 & 50 & 97.5 \\
\hline Total cholesterol & 1.04 & 1.70 & 3.26 & 1.04 & 1.73 & 3.19 & 1.03 & 1.68 & 3.56 & 0.98 & 1.63 & $-{ }^{a}$ \\
\hline Triglycerides & 0.24 & 0.46 & 1.45 & 0.20 & 0.46 & 1.38 & 0.24 & 0.44 & 1.51 & 0.29 & 0.54 & $-{ }^{a}$ \\
\hline
\end{tabular}

All blood lipid concentrations given in $\mathrm{mmol} / \mathrm{L}, B M I$ Body Mass Index $\left(\mathrm{kg} / \mathrm{m}^{2}\right)$

${ }^{\text {a }}$ Sample size insufficient to calculate the 97.5 th percentile

concentrations for triglycerides in early and late pregnancy are both in-line with the recommendations for a nonpregnant cohort, however, while in early pregnancy $3.9 \%$ of the cohort had triglyceride concentrations $>2 \mathrm{mmol} / \mathrm{L}$, this increases to $30 \%$ in late pregnancy. Early pregnancy cholesterol and triglyceride concentrations were not associated with fetal concentrations, however, in late pregnancy, both total cholesterol and triglyceride concentrations were positively associated with fetal total cholesterol and triglyceride concentrations $(P=0.02$ and $P=0.04$, respectively).

There were significant differences in blood lipid concentrations between the BMI categories during pregnancy. In early pregnancy, women in the normalweight category (BMI $<25 \mathrm{~kg} / \mathrm{m}^{2}$ ) had lower concentrations of total cholesterol compared to women in the overweight category (BMI $\left.25-29.9 \mathrm{~kg} / \mathrm{m}^{2}\right)(P=0.02)$. Women in the obese category (BMI $>30 \mathrm{~kg} / \mathrm{m}^{2}$ ) had higher concentrations of triglycerides in early pregnancy than both women in the normal-weight and overweight categories $(P<0.001$ and $P=0.03$, respectively). In late pregnancy, while total cholesterol concentrations didn't differ significantly between the groups, triglyceride concentrations remained elevated in women in the obese category compared to women in the normal-weight category only $(P=0.005)$. There were no differences in triglyceride concentrations between the overweight and obese categories in late pregnancy. There were also no differences between the cord blood lipid concentrations of infants born to mothers in the normal-weight, overweight or obese categories.

Infant size was associated with triglyceride concentrations in late pregnancy in this cohort (see Fig. 1). Triglyceride concentrations were higher in mothers who had a macrosomic baby $(1.84 \mathrm{mmol} / \mathrm{L}$ vs. $1.63 \mathrm{mmol} / \mathrm{L}, P=0.01)$. In cases of LGA infants, triglycerides in late pregnancy were also higher $(1.86 \mathrm{mmol} / \mathrm{L}$ vs. $1.66 \mathrm{mmol} / \mathrm{L}, P=0.025)$. On binary logistic regression, after adjustment for maternal BMI category, triglyceride levels in late pregnancy remained associated with an increased risk of macrosomia (OR: 1.49, CI: 1.05-2.13) and LGA (OR: 1.61, CI: 1.09-2.38). There were no differences in any other blood lipids related to incidence of macrosomia or LGA. Neonatal sex had no association with maternal or fetal lipid concentrations.

\section{Discussion}

Maternal blood cholesterol and triglyceride concentrations increased as pregnancy progressed in this cohort. During pregnancy, maternal blood volume increases by up to $45 \%$ (21) and we observed that maternal lipid concentrations increase above this, resulting in a hyperlipidemic state. In addition, maternal weight was associated with blood lipid concentrations as a higher BMI was associated with higher concentrations of total cholesterol in early pregnancy, and triglycerides in early and late pregnancy.

Our results are in accord with previous studies that have shown that blood lipid concentrations increase during pregnancy, with cholesterol concentrations estimated to increase by up to $70 \%$ compared to pre-pregnancy concentrations $[3,16,22-24]$. Compared to nonpregnant women, triglyceride concentrations in late pregnancy may be up to $138 \%$ higher [3]. While blood lipid concentrations after pregnancy were not available in this cohort, other research has indicated that lipid concentrations return to pre-pregnancy concentrations after delivery, suggesting that the increase in blood lipids could have an important role in the development of the 


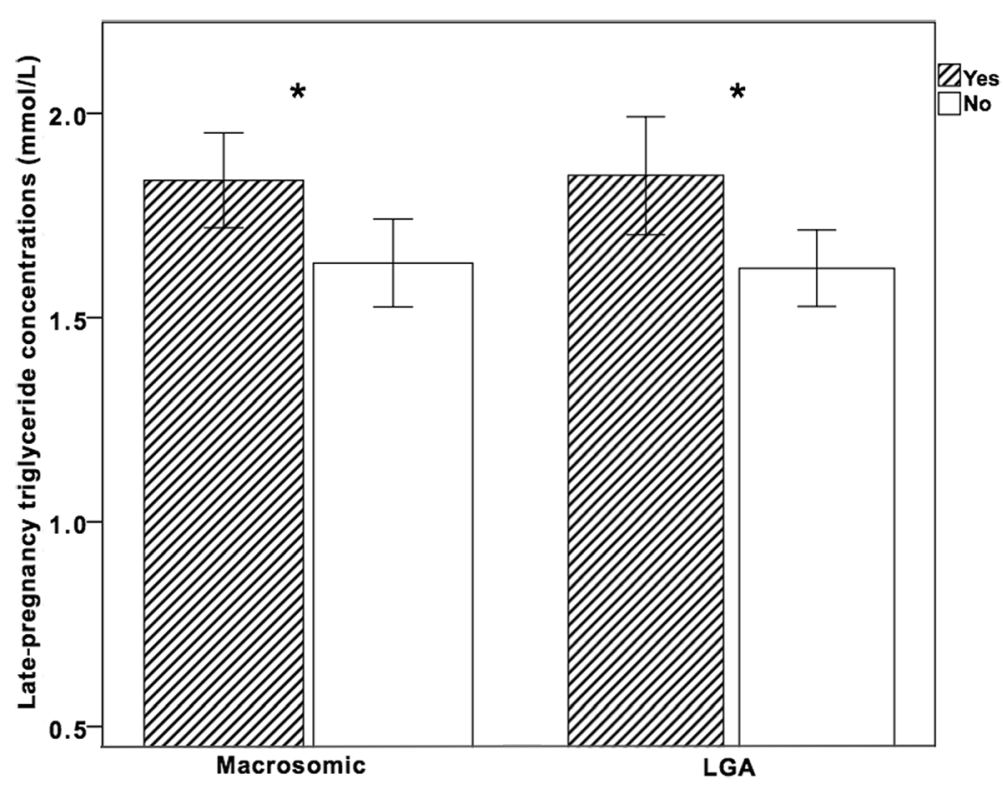

Fig. 1 Maternal triglyceride concentrations in late pregnancy in macrosomic neonates ( $>4 \mathrm{~kg}$ weight) or large-for-gestational-age ( $>90$ th centile)

fetus [3, 23, 24]. This is further supported by research highlighting that the increase in blood lipids during pregnancy is not associated with an increased risk of atherosclerosis in women [3, 25]. In fact, raised HDL-C may have a role in protecting the maternal vascular endothelium during pregnancy [26, 27]. It is thought that a failure of the body to increase HDL-C concentrations during pregnancy may be linked with the development of pre-eclampsia [27].

The differences we observed in triglyceride concentrations between the BMI categories could be an indication of altered metabolism or possibly increased levels of insulin resistance in pregnant women with overweight or obesity [28]. Previous research in this cohort reported an association between triglyceride concentrations and birth weight in women in the overweight or obese category only [29]. This highlights the importance of carrying out further research to establish normal lipid concentrations in pregnancy as these concentrations could indicate future risk for the women or offspring. In our cohort there were no differences in fetal lipid concentrations between the BMI groups. Given that maternal concentrations, but not fetal blood lipid concentrations, differed by maternal BMI, this raises an interesting question as to the role of the placenta as a potential mediator. Research has shown that the composition of particular lipoproteins may influence the flux of lipids to the placenta which may play a mechanistic role here [30]. Further research on lipid concentrations in fetal blood may be required to establish normal levels and the physiological implications of abnormal values.
Other studies have reported different blood lipid profiles and metabolic responses in mothers in the normal-weight and overweight or obese categories [17]. One such study found that women in the overweight or obese category had higher baseline cholesterol and triglyceride concentrations compared to women in the normal-weight category; however, in later pregnancy the women in the normal-weight category had higher total cholesterol concentrations as their weekly concentrations increased at a faster rate during pregnancy [16]. This is comparable to our findings where women in the normal-weight and overweight categories had similar total cholesterol concentrations in late pregnancy, despite higher concentrations among overweight/obese categories in early pregnancy. Recently published research has also highlighted how energy metabolism during pregnancy differs between women in overweight and normal-weight BMI categories [30]. During pregnancy women with a BMI $>25$ had higher lipid oxidation rates than women with a BMI $<25$, particularly at the end of pregnancy [30]. This may also explain why our total cholesterol levels in late pregnancy are more similar between the two groups as the mothers in the overweight category may have had higher lipid oxidation rates at this stage. Published reports describing maternal blood lipids in pregnancy have largely focused on women with gestational diabetes or glucose intolerance $[5,6]$. To date, studies relating alterations in blood lipid concentrations during nondiabetic pregnancy have had sample sizes below the 120 recommended by the Clinical and Laboratory Standards Institute [31], or have used values based on just one time point $[8,22,23]$. 
This analysis had many strengths in that there was a large number of participants in the total study and blood samples were collected in both early and late pregnancy, which can help us understand normal changes over the course of a pregnancy. Blood lipid profiles were also analysed in fetal blood and there is currently a paucity of data in this area. To our knowledge, this is one of the few studies to create lipid reference intervals during pregnancy and to categorise them by BMI, which adds to this body of literature. There were some limitations that must be taken into account. In this cohort, 54.7\% of the babies were classified as macrosomic and $29.3 \%$ as LGA so this may not be representative of the entire population, however these mothers were healthy with no pregnancy complications. There were also a small number of participants in the obese category (BMI $\geq 30 \mathrm{~kg}$ / $\mathrm{m}^{2}$ ), thus the 97.5th percentile could not be calculated for the fetal cord samples in this group.

With maternal blood lipid profiles in pregnancy being associated with both maternal and child outcomes, it may be advisable to monitor blood lipid concentrations in pregnant women to identify both normal concentrations during pregnancy and the levels at which lipid concentrations become problematic. This could potentially be used to identify concentrations associated with increased risks of adverse outcomes in pregnancy, particularly abnormal fetal growth, and allow for early interventions to prevent negative outcomes for the mother or infant. Given that there was no difference in fetal concentrations between the BMI categories but there was in maternal concentrations, the potential mediating role of the placenta and the influence of blood lipid composition in limiting lipid transfer also remains to be explored.

\section{Conclusion}

Maternal blood lipid concentrations increase over the course of pregnancy and the concentrations at which this can negatively impact both the health of the mother and the child remains to be elucidated. We described intervals in healthy women for total cholesterol and triglyceride concentrations during pregnancy, and also in fetal cord blood. We found that maternal cholesterol and triglyceride concentrations differ by BMI category which may indicate altered metabolic functioning. Blood lipid concentrations are associated with negative outcomes for the mother and infant, thus these intervals can help to inform future references for blood lipid concentrations throughout pregnancy.

\section{Abbreviations}

BMl: Body Mass Index; Cl: Confidence Intervals; HDL-C: High-density Lipoproteins; LGA: Large-for-gestational-age; OR: Odds Ratio

\section{Funding}

This study was supported by the Health Research Board Ireland, the Health Research Centre for Health and Diet Research, The National Maternity Hospital Medical Fund and the European Union's Seventh Framework Programme (FP7/2007-2013), project EarlyNutrition under grant agreement no. 289346

\section{Availability of data and materials}

The datasets used and/or analysed during the current study available from the corresponding author on reasonable request.

\section{Authors' contributions}

AAG, FMMA and PJT conceived the idea and were responsible for the study design. AAG, BC and FMMA obtained the data. AAG, GA, EJOS and ECOB analysed and interpreted the data and AAG carried out the statistical analysis. AG wrote the original draft and FMMA, GA, EJOS and ECOB critically reviewed and edited the manuscript. All authors read and approved the final manuscript

\section{Ethics approval and consent to participate}

This study was carried out with ethical approval from the National Maternity Hospital Ethics Committee, Dublin and maternal written consent was obtained.

Consent for publication

Not applicable.

\section{Competing interests}

The authors declare that they have no competing interests.

\section{Publisher's Note}

Springer Nature remains neutral with regard to jurisdictional claims in published maps and institutional affiliations.

\section{Author details}

${ }^{1}$ UCD Perinatal Research Centre, Obstetrics and Gynaecology, School of Medicine, University College Dublin, National Maternity Hospital, Dublin 2, Ireland. ${ }^{2}$ Clinical Chemistry, St. Vincent's University Hospital, Dublin 4, Ireland. ${ }^{3}$ UCD School of Medicine, University College Dublin, Dublin, Ireland.

Received: 27 January 2017 Accepted: 8 October 2017

Published online: 16 October 2017

References

1. Mazurkiewicz JC, Watts GF, Warburton FG, Slavin BM, Lowy C, Koukkou E. Serum lipids, lipoproteins and apolipoproteins in pregnant non-diabetic patients. J Clin Pathol. 1994;47:728-31. https://doi.org/10.1136/jcp.47.8.728.

2. Herrera E, Ortega-Senovilla H. Lipid metabolism during pregnancy and its implications for fetal growth. Curr Pharm Biotechnol. 2014:24-31. https:// doi.org/10.2174/1389201015666140330192345.

3. An-Na C, Man-Li Y, Jeng-Hsiu H, Pesus C, Shin-Kuo S, Heung-Tat N. Alterations of serum lipid levels and their biological relevances during and after pregnancy. Life Sci. 1995;56:2367-75. https://doi.org/10.1016/00243205(95)00230-4.

4. Wang C, Zhu W, Wei Y, Su R, Feng H, Lin L, et al. The predictive effects of early pregnancy lipid profiles and fasting glucose on the risk of gestational diabetes mellitus stratified by body mass index. Journal of Diabetes Research. 2016;2016:3013567. https://doi.org/10.1155/2016/3013567.

5. Enquobahrie DA, Williams MA, Qiu C, Luthy DA. Early pregnancy lipid concentrations and the risk of gestational diabetes mellitus. Diabetes Res Clin Pract. 2005;70:134-42. https://doi.org/10.1016/j.diabres.2005.03.022.

6. Ryckman KK, Spracklen CN, Smith CJ, Robinson JG, Saftlas AF. Maternal lipid levels during pregnancy and gestational diabetes: a systematic review and meta-analysis. BJOG An Int J Obstet Gynaecol. 2015;122:643-51. https://doi. org/10.1111/1471-0528.13261.

7. Vrijkotte TGM, Krukziener N, Hutten BA, Vollebregt KC, Van Eijsden M, Twickler MB. Maternal lipid profile during early pregnancy and pregnancy complications and outcomes: the ABCD study. J Clin Endocrinol Metab. 2012;97:3917-25. https://doi.org/10.1210/jc.2012-1295.

8. Winkler K, Wetzka B, Hoffmann MM, Friedrich I, Kinner M, Baumstark MW, et al. Low density lipoprotein (LDL) subfractions during pregnancy: 
accumulation of buoyant LDL with advancing gestation. J Clin Endocrinol Metab. 2000;85:4543-50. https://doi.org/10.1210/jcem.85.12.7027.

9. Schaefer-Graf UM, Graf K, Kulbacka I, Kjos SL, Dudenhausen J, Vetter K, et al. Maternal lipids as strong determinants of fetal environment and growth in pregnancies with gestational diabetes mellitus. Diabetes Care. 2008;31: 1858-63. https://doi.org/10.2337/dc08-0039.

10. Kulkarni SR, Kumaran K, Rao SR, Chougule SD, Deokar TM, Bhalerao AJ, et al. Maternal lipids are as important as glucose for fetal growth: findings from the pune maternal nutrition study. Diabetes Care. 2013;36:2706-13. https:// doi.org/10.2337/dc12-2445.

11. Gobl C, Handisurya A, Klein K, Bozkurt L, Luger A, Bancher-Todesca D, et al. Changes in serum lipid levels during pregnancy in type 1 and type 2 diabetic subjects. Diabetes Care. 2010;33:9-11. https://doi.org/10.2337/dc10-0484.

12. Leiva A, Salsoso R, Saez T, Sanhueza C, Pardo F, Sobrevia L. Cross-sectional and longitudinal lipid determination studies in pregnant women reveal an association between increased maternal LDL cholesterol concentrations and reduced human umbilical vein relaxation. Placenta. 2015;36:895-902. https:// doi.org/10.1016/j.placenta.2015.05.012.

13. Son GH, Kwon JY, Kim YH, Park YW. Maternal serum triglycerides as predictive factors for large-for-gestational age newborns in women with gestational diabetes mellitus. Acta Obstet Gynecol Scand. 2010;89:700-4. https://doi.org/10.3109/00016341003605677.

14. Kwiterovich PO, Cockrill SL, Virgil DG, Garrett ES, Otvos J, Knight-Gibson C, et al. A large high-density lipoprotein enriched in apolipoprotein C-I. JAMA. 2005;293:1891-9. https://doi.org/10.1001/jama.293.15.1891.

15. Jellinger PS, Smith DA, Mehta AE, Ganda O, Handelsman Y, Rodbard HW, et al. American association of clinical endocrinologists' (AACE) guidelines for management of dyslipidemia and prevention of atherosclerosis. Endocr Pract. 2012;18:1-35. https://doi.org/10.4158/EP.17.S2.1.

16. Scifres $\mathrm{CM}$, Catov JM, Simhan HN. The impact of maternal obesity and gestational weight gain on early and mid-pregnancy lipid profiles. Obesity (Silver Spring). 2014;22:932-8. https://doi.org/10.1002/oby.20576.

17. Vahratian A, Misra VK, Trudeau S, Misra DP. Prepregnancy body mass index and gestational age-dependent changes in lipid levels during pregnancy. Obstet Gynecol. 2010;116:107-13. https://doi.org/10.1097/AOG. 0b013e3181e45d23.

18. McGowan CA, Walsh JM, Byrne J, Curran S, McAuliffe FM. The influence of a low glycemic index dietary intervention on maternal dietary intake, glycemic index and gestational weight gain during pregnancy: a randomized controlled trial. Nutr J. Nutr J. 2013;12:140. https://doi.org/10. 1186/1475-2891-12-140.

19. Horan MK, McGowan CA, Gibney ER, Donnelly JM, McAuliffe FM. Maternal low glycaemic index diet, fat intake and postprandial glucose influences neonatal adiposity - secondary analysis from the ROLO study. Nutr J. 2014; 13:78. https://doi.org/10.1186/1475-2891-13-78

20. Horan MK, McGowan CA, Gibney ER, Donnelly JM, McAuliffe FM. Maternal diet and weight at 3 months postpartum following a pregnancy intervention with a low glycaemic index diet: results from the ROLO randomised control trial. Nutrients. 2014;6:2946-55. https://doi.org/10.3390/nu6072946.

21. Walsh JM, McGowan CA, Mahony R, Foley ME, McAuliffe FM. Low glycaemic index diet in pregnancy to prevent macrosomia (ROLO study): randomised control trial. BMJ. 2012;345:e5605. https://doi.org/10.1136/bmj.e5605.

22. Lippi G, Albiero A, Montagnana M, Salvagno GL, Scevarolli S, Franchi M, et al. Lipid and lipoprotein profile in physiological pregnancy. Clin Lab. 2007; 53(3-4):173-7.

23. Bartels Ä, Egan N, Broadhurst DI, Khashan AS, Joyce C, Stapleton M, et al. Maternal serum cholesterol levels are elevated from the 1st trimester of pregnancy: a cross-sectional study. J Obstet Gynaecol. 2012;32:747-52. https://doi.org/10.3109/01443615.2012.714017.

24. Husain F, Latif S, Uddin M. Studies on serum total cholesterol, in second and third trimester of pregnancy. J Bangladesh Soc Physiol. 2006 Dec;1:1-4.

25. Neboh E, Emeh J, Aniebue U, Ikekpeazu E, Maduka I, Ezeugwu F. Relationship between lipid and lipoprotein metabolism in trimesters of pregnancy in Nigerian women: is pregnancy a risk factor? J Nat Sci Biol Med. 2012;3:32-7. https://doi.org/10.4103/0976.

26. Sulaiman WNW, Caslake MJ, Delles C, Karlsson H, Mulder MT, Freeman DJ. Does high-density lipoprotein protect vascular function in healthy pregnancy? Clincal Sci. 2016;130:491-7. https://doi.org/10.1042/CS20150475.

27. Barrett HL, Dekker Nitert M, McIntyre HD, Callaway LK. Maternal lipids in preeclampsia: innocent bystander or culprit? Hypertens Pregnancy. 2014;33: 508-23. https://doi.org/10.3109/10641955.2014.946614.
28. Catalano PM. Obesity, insulin resistance, and pregnancy outcome. Reproduction. 2010;140:365-71. https://doi.org/10.1530/REP-10-0088,

29. Geraghty AA, Alberdi G, O'Sullivan EJ, O'Brien EC, Crosbie B, Twomey PJ, et al. Maternal blood lipid profile during pregnancy and associations with child adiposity: findings from the ROLO study. PLoS One. 2016;11:1-13. https:// doi.org/10.1371/journal.pone.0161206.

30. Bugatto F, Quintero-Prado R, Vilar-Sánchez JM, Perdomo G, Torrejón R, Bartha JL. Prepregnancy body mass index influences lipid oxidation rate during pregnancy. Acta Obstet Gynecol Scand. 2017;96:207-15. https://doi. org/10.1111/aogs.13058.

31. Appold K. Determining laboratory reference intervals: CLSI guideline makes the task manageable. Lab Med. 2009;40:75-6. https://doi.org/10.1309/ LMEHV3HP39Q0FJPA.

\section{Submit your next manuscript to BioMed Central and we will help you at every step:}

- We accept pre-submission inquiries

- Our selector tool helps you to find the most relevant journal

- We provide round the clock customer support

- Convenient online submission

- Thorough peer review

- Inclusion in PubMed and all major indexing services

- Maximum visibility for your research

Submit your manuscript at www.biomedcentral.com/submit
) Biomed Central 